\title{
छூ \\ High power fiber laser system for a high repetition rate laserwire
}

\author{
L. J. Nevay, ${ }^{*}$ R. Walczak, and L. Corner \\ John Adams Institute, University of Oxford, Denys Wilkinson Building, Keble Road, \\ Oxford OX1 3RH, United Kingdom
}

(Received 1 October 2013; published 9 July 2014)

\begin{abstract}
We present the development of a high power fiber laser system to investigate its suitability for use in a transverse electron beam profile monitor, i.e., a laserwire. A system capable of producing individual pulses up to $165.8 \pm 0.4 \mu \mathrm{J}$ at $1036 \mathrm{~nm}$ with a full width at half maximum of $1.92 \pm 0.12 \mathrm{ps}$ at $6.49 \mathrm{MHz}$ is demonstrated using a master oscillator power amplifier design with a final amplification stage in a rod-type photonic crystal fiber. The pulses are produced in trains of $1 \mathrm{~ms}$ in a novel burst mode amplification scheme to match the bunch pattern of the charged particles in an accelerator. This method allows pulse energies up to an order of magnitude greater than the steady-state value of $17.0 \pm 0.6 \mu \mathrm{J}$ to be achieved at the beginning of the burst with a demonstrated peak power of $25.8 \pm 1.7 \mathrm{MW}$ after compression. The system is also shown to demonstrate excellent spatial quality with an $M^{2}=1.26 \pm 0.01$ in both dimensions, which would allow nearly diffraction limited focusing to be achieved.
\end{abstract}

DOI: 10.1103/PhysRevSTAB.17.072801

PACS numbers: 29.20.Ej, 29.27.Fh, 42.55.Wd

\section{INTRODUCTION}

For a future linear electron-positron collider such as the International Linear Collider (ILC) [1] and the Compact Linear Collider (CLIC) [2], the generation and transport of beams while preserving the low emittance is essential to achieve the required final focus beam sizes and therefore high luminosity. This requires measurement, monitoring and control of the transverse beam emittance along the entire accelerator chain, preferably in a noninvasive manner. In a linear accelerator, one possible method of measuring the emittance is by measuring the transverse size of the beam at several points in the lattice with different betatron phases. Two methods to achieve this are wire scanners that measure a projection of the beam and optical transition radiation screens to image the beam directly [3], but these can both suffer damage from high charge density beams. Conventional methods such as these are disruptive to the beam and they cannot be used for measurement and tuning of the accelerator during operation, therefore the development of new diagnostics is essential.

A laserwire is a beam size diagnostic in which a tightly focused laser beam is scanned perpendicularly across an electron beam [4]. A small fraction of the laser photons undergo Compton scattering, resulting in electrons with degraded energies and high energy photons that travel nearly parallel to the electron beam. A dipole magnet

\footnotetext{
*Present address: Royal Holloway, University of London, Surrey TW20 0EX, United Kingdom.

laurie.nevay@rhul.ac.uk

Published by the American Physical Society under the terms of the Creative Commons Attribution 3.0 License. Further distribution of this work must maintain attribution to the author $(s)$ and the published article's title, journal citation, and DOI.
}

further along the accelerator separates these from the primary electron beam as shown in Fig. 1. The Comptonscattered photons can be detected further along the beam line after a bending magnet where the photons and electrons are separated. Alternatively, in the case of a high energy collider $\left(E_{e}>50 \mathrm{GeV}\right)$, the energy of the electrons is degraded by a large fraction and it would be possible to detect these electrons as they are lost from the lattice. Both can be used as a measure of the Compton-scattering rate, which is modulated as the laser focus is scanned transversely across the electron beam, providing a laserwire scan. With knowledge of the laser size at its focus, the scan can be deconvolved to yield the electron beam size [5].

For accurate determination of the electron beam size, the laser spot size must be of a similar or ideally smaller size than the electron beam. The laser must be of sufficient intensity to produce a detectable number of Comptonscattered photons relative to the detector background environment. Furthermore, it must have the excellent spatial beam quality required to create the focused spot sizes of approximately a micrometer that will be required for use in a laserwire at the ILC or CLIC. If a pulsed laser source is used, it must also have pulses of a similar duration to that of the individual electron bunches for optimal laserelectron collision luminosity, which would for example imply a pulse duration of approximately $1 \mathrm{ps}$ for the ILC.

High power commercially available lasers typically emit light in the near infrared. As diffraction limits the achievable focused spot size to approximately the wavelength of the light, it is necessary to convert the laser light to a shorter wavelength to produce the small beam sizes required for a laserwire. However, light with a wavelength shorter than $300 \mathrm{~nm}$ experiences strong absorption in most optical materials necessitating reflective focusing optics rather 


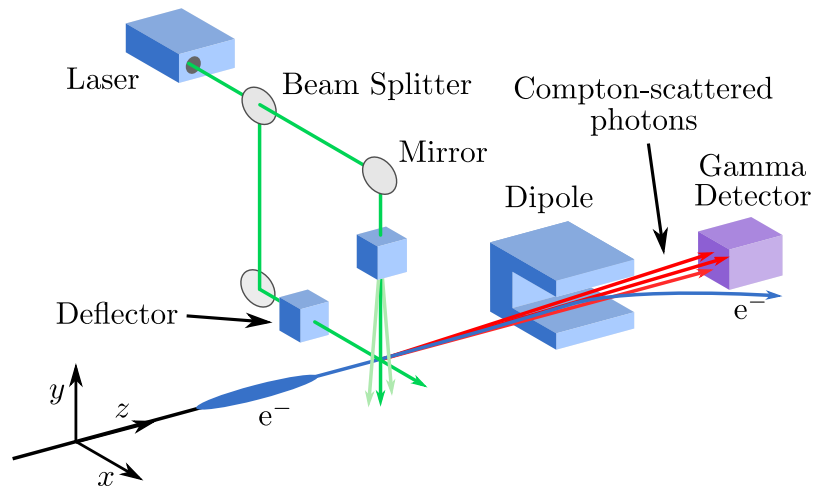

FIG. 1. Conceptual schematic of a laserwire. A laser beam is scanned perpendicularly to an electron beam producing Compton-scattered photons that are detected after a dipole magnet.

than transmissive ones. A laserwire at the Stanford Linear Collider demonstrated micrometer-size scans with submicrometer resolution using an ultraviolet laser with a reflective focusing geometry $[6,7]$. This geometry prevents direct measurement of the laser focus and therefore calibration as well as imposing a very short scanning range due to the optical aberrations incurred when the focusing mirror is used off-axis-for example when either the laser or the mirror is moved to scan the focused laser spot across the electron beam. Transmissive optics afford a much greater scanning range and allow direct measurement of the focused spot size. Given these advantages, a compromise is to use a visible wavelength generated by frequency doubling a near infrared source, which increases the resolution but reduces the peak power [8].

Apart from the laserwire at Stanford Linear Collider, several other laserwires have been demonstrated. A laserwire at PETRA-III in DESY [9] using a commercial Q-switched pulsed laser at $20 \mathrm{~Hz}$ made laserwire scans of $\sigma \sim 30 \mu \mathrm{m}$ on electron bunches in a ring accelerator with a precision of 5\%. An installation at the damping ring of the Accelerator Test Facility 2 used a continuous-wave laser with cavity enhancement to measure an electron beam size of $9.8 \mu \mathrm{m}$ [10]. Here, the very low Compton-scattering rate from the low power laser is balanced by the megahertz revolution frequency of the electron bunches in the ring. In these cases, the low repetition rate and low average laser power respectively preclude fast intratrain scanning as would be required at the ILC. Another laserwire installation at the linear extraction line of Accelerator Test Facility 2 has shown micrometer-sized scans using a gigawatt peak power $Q$-switched pulsed laser at $3.25 \mathrm{~Hz}$ [11]. Despite the high resolution and signal level at this installation, the low repetition rate of the laser system also prevents intratrain scanning. In each case, the laser technology limits the usability of the laserwire as a diagnostic for a future linear collider.

In this paper, we consider the requirements for a laserwire at the ILC and present the results of a fiber laser system developed for use there. Its suitability is demonstrated through the calculation of the yield of Comptonscattered photons for such a system at the ILC.

\section{ILC LASERWIRE SPECIFICATIONS}

The ILC is a proposed linear collider that is intended to collide electrons and positrons initially at $250 \mathrm{GeV}$ and eventually up to $500 \mathrm{GeV}$. Such a machine is needed to make precision studies of the recently discovered Higgs boson and to investigate potential new discoveries at the Large Hadron Collider at CERN and will require 20 laserwires in various locations as a primary accelerator diagnostic [1]. Laserwires will be of crucial importance in the beam delivery system (BDS) after the main linac, where precise measurements of the electron beam size of the order of a micrometer are necessary to ensure that the electron and positron beams can be focused to the required nanometer sizes at the interaction point. The parameters of the electron beam in the ILC BDS are shown in Table I.

The luminosity of the laser photon and electron collision is linearly proportional to both the number of photons and the number of electrons as well as the cross section for the interaction, which in this case is the Compton cross section. Agapov et al. [5] have shown that given typical detector efficiencies and operational experience of a laserwire, a wavelength of $532 \mathrm{~nm}$, laser pulse length of $\sigma_{\tau}=1 \mathrm{ps}$ and focused to a size of $\sigma_{l}=1 \mu \mathrm{m}$, that a laser peak power of approximately $10 \mathrm{MW}$ will be required for each of the laserwires planned for use in the ILC. Currently available commercial laser systems capable of this peak power can do so only at kilohertz repetition rates and typically have a low overall efficiency. Such a system would therefore preclude intratrain scanning at megahertz repetition rates, and given the low electron bunch train repetition rate of $5 \mathrm{~Hz}$, would take much longer to achieve the required measurement precision. In the case of a future linear collider, the emittance measurement system may be used often for continuous feedback and tuning purposes and a system utilizing intratrain scanning would decrease the measurement time as well as increase the precision. Additionally, the spatial quality of such laser systems

TABLE I. Nominal ILC BDS electron beam parameters.

\begin{tabular}{lccc}
\hline \hline Parameter & Symbol & Value & Units \\
\hline Beam energy & $\mathrm{E}$ & 250 & $\mathrm{GeV}$ \\
Normalized horizontal emittance & $\epsilon_{x}^{*}$ & 10 & $\mu \mathrm{m} \mathrm{rad}$ \\
Normalized vertical emittance & $\epsilon_{y}^{*}$ & 40 & $\mathrm{~nm} \mathrm{rad}$ \\
Train repetition frequency & $f_{\text {train }}$ & 5 & $\mathrm{~Hz}$ \\
Number of bunches per train & $N_{\text {train }}$ & 1312 & \\
Bunch repetition frequency & $f_{\text {bunch }}$ & 1.81 & $\mathrm{MHz}$ \\
Bunch duration & $\sigma_{\tau e}$ & 1 & $\mathrm{ps}$ \\
Number of electrons per bunch & $N_{e}$ & $2 \times 10^{10}$ & \\
\hline \hline
\end{tabular}


can be unsuitable for achieving the necessary diffractionlimited focused spot sizes.

A peak power of $10 \mathrm{MW}$ with a pulse length of $1 \mathrm{ps}$ corresponds to an energy of $10 \mu \mathrm{J}$ per pulse. High energy ultrashort laser pulses in the visible part of the spectrum are typically produced by frequency doubling a source at $\lambda \sim 1 \mu \mathrm{m}$, which can be achieved with an efficiency of $60 \%$ [12]. Therefore, pulses of $\sim 17 \mu \mathrm{J}$ in the near infrared are required at the megahertz repetition rate to allow intratrain scanning of the electron bunches.

\section{FIBER LASER}

A technology with great potential for laserwires and other accelerator applications is that of optical fiber lasers, which has progressed rapidly in recent years, allowing the efficient generation of high energy ultrashort pulses [13]. The waveguide structure of optical fibers means they have excellent spatial quality and high efficiency due to the long interaction lengths with the pump sources. However, high intensities that arise in the small core diameters of single mode step-index fibers can induce optical nonlinearities that affect the spectral and temporal properties of the output pulses and limit the maximum useful output [14]. One solution to this problem has been the development of photonic crystal fibers (PCFs) that have large mode areas with nearly single mode spatial output that allow high energy pulse extraction [15]. In particular, rod-type PCFs have the largest core diameters that can be up to $100 \mu \mathrm{m}$ [16] and have been shown to produce $1 \mathrm{~mJ}, 800 \mathrm{fs}$ output pulses at $100 \mathrm{kHz}$ [17]. Rod-type PCFs cannot be connectorized like normal optical fibers, but they are desirable as their large area allows the highest peak powers to be achieved without optical nonlinearities. Fiber lasers have excellent conversion of pump to laser light and, in addition, are pumped by highly efficient diode sources leading to very high overall wall plug efficiency. This, together with the excellent spatial quality and reliability of fiber lasers make them attractive for applications such as laserwire.

To investigate the suitability of a fiber laser for a laserwire, a system was developed consisting of a commercial chirped pulse amplification fiber laser followed by further amplification in an $80 \mathrm{~cm}$ long $\mathrm{Yb}$-doped rod-type PCF (NKT Photonics DC-200/85-Yb-ROD). The commercial laser (Amplitude Systèmes) consists of a Yb:KYW oscillator at $1036 \mathrm{~nm}$ producing $19 \mathrm{~nJ}$ pulses at $51.92 \mathrm{MHz}$, locked to a $\mathrm{Rb}$ stabilized external radio frequency signal generator. The 500 fs oscillator pulses are stretched to $\sim 200 \mathrm{ps}$ and then the repetition rate reduced to $6.49 \mathrm{MHz}$ by an acousto-optic modulator. The pulses (now $6 \mathrm{~nJ}$ ) are amplified in two stages in Yb-doped $25 \mu \mathrm{m}$ core diameter fiber and then pass through an electro-optic modulator (EOM) for macropulse shaping, producing $\sim 1.5 \mu \mathrm{J}$ output pulses in bursts as short as $500 \mathrm{~ns}$, which are used as a seed for the PCF.

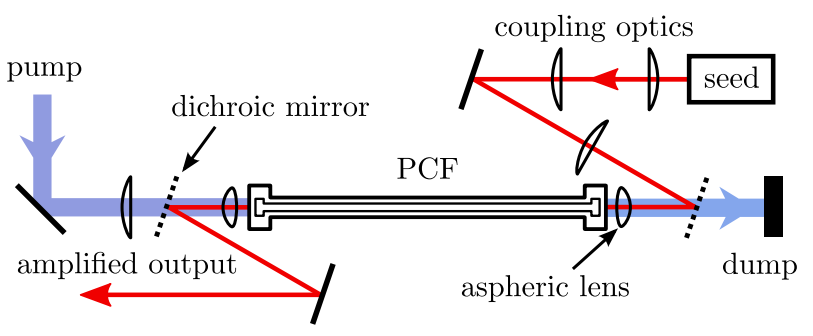

FIG. 2. Experimental arrangement for amplification in a PCF with coupling and decoupling aspheric lenses as well as dichroic mirrors to separate the pump and seed beams.

The PCF has a core diameter of $85 \mu \mathrm{m}$ to guide the seed, an inner cladding diameter of $200 \mu \mathrm{m}$ to guide the pump, and an outer cladding diameter of $1.7 \mathrm{~mm}$. The $400 \mathrm{~W}$, $976 \mathrm{~nm}$ pump diode laser (Newport Spectra-Physics) is coupled into the PCF in a counterpropagating geometry allowing independent optimization of both the seed and the pump coupling as well as increased efficiency [18]. Fused silica end caps $8 \mathrm{~mm}$ long and $10 \mathrm{~mm}$ diameter at both ends of the fiber allow the unguided light from the core and inner cladding to expand before exiting through the glass air interface, reducing the possibility of damage to the fiber [19]. The seed and pump are separated by dichroic mirrors at either end of the PCF, which is made entirely of silica with no polymer coating and is supported in a metal $\mathrm{V}$ groove. Due to the high efficiency of fiber lasers and the large surface area to volume ratio, the fiber requires neither active nor passive cooling. The experimental arrangement is shown in Fig. 2.

\section{A. Burst mode amplification}

Whilst high peak power laser pulses are required at megahertz repetition rates to match the electron beam for intratrain scanning, the bunch trains have a low duty cycle $(0.4 \%)$. The laser system was matched to produce pulses with a similar duty cycle, significantly reducing the average power and therefore cooling requirements as well as avoiding wasting laser energy when there are no electron bunches. The EOM in the seed laser allows the necessary macropulse shaping and the pump output is modulated using the output of a signal generator as a gate to the power supply.

Simultaneously starting both the pump and the seed will not result in a uniform burst of amplified pulses as the pump has a finite rise time $(\sim 100 \mu \mathrm{s})$. With a simultaneous start, the PCF will not be pumped initially and the $\mathrm{Yb}$ dopant will absorb the incoming seed due to its quasi-threelevel nature. During the rise time of the pump, the PCF becomes first transparent and then amplifies the seed. By turning on the pump before the seed by approximately the rise time of the pump a more uniform output can be achieved. However, by further increasing the delay of the seed burst with respect to the pump the $\mathrm{Yb}$ dopant is 


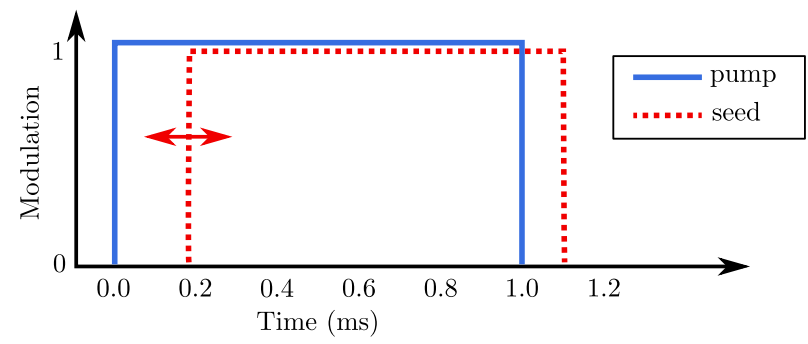

FIG. 3. Modulation envelopes of the seed and pump laser for burst operation with the pump preceding the seed for high gain.

pumped without the seed depleting its excited upper-state population and energy can be stored in the PCF resulting in higher gain during the seed burst. After approximately the upper state lifetime of $\mathrm{Yb}$ in glass $(0.8 \mathrm{~ms})$, spontaneous emission of the excited population will reduce the available gain and may lead to large laser pulses oscillating in the rod that can lead to catastrophic damage [20].

An investigation was carried out to characterize this process and in doing so a method was demonstrated by which pulses almost an order of magnitude greater in energy than the steady state can be achieved. The pump laser requires a minimum operation time of $1 \mathrm{~ms}$, which corresponds to $\sim 6500$ pulses of the seed. Initially, the seed and pump bursts were operated simultaneously and then the start of the seed burst was delayed with respect to the start of the pump as shown in Fig. 3. As the pump laser also has a finite fall time and some excited upper-state population still exists even when the pump is turned off, the seed burst was extended by $100 \mu \mathrm{s}$ beyond the end of the pump to safely extract the stored energy. The system was operated at $2 \mathrm{~Hz}$ due to the limitation of the energy meter used to integrate the energy of the full burst and the system is capable of operating at higher burst repetition rates.

As this technique relies on a buildup of gain beyond the normal steady state, it is to be expected that the high repetition rate seed pulses will deplete the upper-state population before it is replenished by the pump. Consequently, the initial pulses in the burst will experience a very high gain with subsequent pulses experiencing decreasing gain until the steady state is reached. Although it is possible to compensate for this by modulating the incoming seed train before the PCF, this is still an active area of research, and can lead to lower individual pulse energies in the burst [21]. Despite the lack of uniformity in pulse energies, this scheme serves to investigate the potential output from a fiber laser and could also be compensated for by pulse energy normalization in a laserwire system.

\section{B. Characterization}

To characterize the output of the laser system, the pulse energies, spatial quality and temporal profiles were measured. The megahertz repetition rate of the pulses presents many practical challenges for conventional techniques used to measure these aspects of the pulses on an individual basis.

\section{Pulse energies}

The amplified seed burst from the PCF with a seed delay relative to the pump of $0.13 \mathrm{~ms}$ is depicted in a photodiode trace in Fig. 4, which shows a high initial series of pulses that rapidly decay to a steady-state level. As the pulses vary in energy significantly they cannot be assumed to be the same and must be measured individually. The megahertz repetition rate is beyond the ability of the fastest commercially available energy meters to discriminate individual pulses. Therefore, an energy meter capable of measuring the energy of the total burst (Gentec QE25-SM-LP) was used in combination with a digitized trace of a fast $(<35 \mathrm{ps}$ rise time) photodiode (Newport 818-BB-25 InGaAs) to resolve the individual pulse energies. As the peak of the photodiode pulses is proportional to the laser pulse energy, the digitized photodiode signal can be used to subdivide the total energy of the burst. The energy of a given pulse in the burst is therefore given by

$$
E_{(i)}=\left(\frac{E_{\text {total }}}{\sum_{i} V_{\text {peak }(i)}}\right) V_{\text {peak }(i)},
$$

where $E_{(i)}$ and $V_{\text {peak }(i)}$ are the pulse energy and peak voltage of the $i$ th pulse respectively, and $E_{\text {total }}$ is the total energy of the burst. An oscilloscope with a high bandwidth, sample rate and sample memory was used (LeCroy WaveRunner 402-MXi) to digitize the photodiode trace of the total burst with 12 bit precision at 10 gigasamples $\mathrm{s}^{-1}$. Two Fresnel beam splitters were used with several neutral density filters to reduce the intensity of the pulses to within the measured linear range of the photodiode and ten waveforms recorded for statistical purposes.

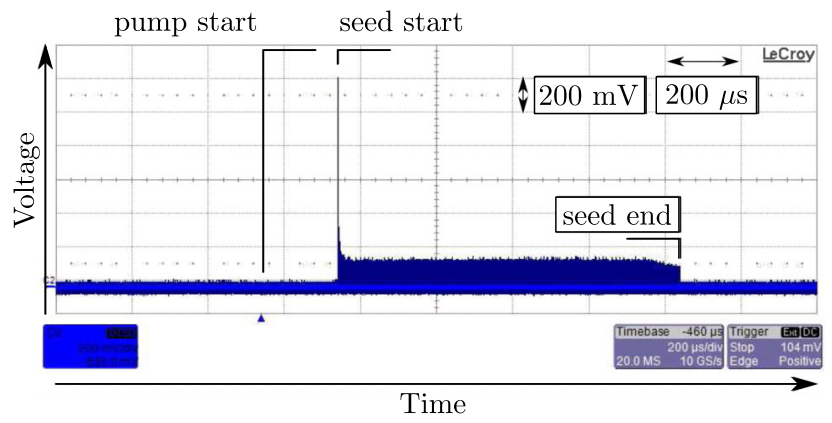

FIG. 4. Oscilloscope screenshot showing a 20 megasample photodiode trace of the amplified output exhibiting exponential decay of pulse energies to a steady-state level. The high number of pulses in the $\sim 1 \mathrm{~ms}$ burst results in a solid trace when viewed on this time scale and the aliasing of the oscilloscope displays a much larger degree of noise than is actually present. 
For the most accurate measurement of the pulse energies in the seed burst, the photodiode traces were recorded using two different scalings on the oscilloscope; first, with a scaling such that the high voltage peaks of the initial laser pulses were sampled properly; and, second, scaled so that the initial pulses were clipped but the majority of the burst was digitized with a higher precision. This method of using two combined sets of photodiode traces reduces the uncertainty in the calculated pulse energy from approximately $20 \%$ to $1 \%$, which is due to the sum of the peak voltage of several thousand pulses. Additionally, the extension of the seed beyond the pump was chosen to give a discrete end to the pulse train making the number of pulses in the burst clearly identifiable.

The pump level was increased until sharp spikes appeared in the amplified spectrum - a sign of the presence of amplified spontaneous emission and an indication that additional pump energy would not produce further useful amplified output. The incident pump burst energy was $297 \pm 1 \mathrm{~mJ}$ and the input seed burst energy was $6.43 \pm 0.02 \mathrm{~mJ}$, which corresponds to an input pulse energy of $0.95 \pm 0.01 \mu \mathrm{J}$. A seed delay of $200 \mu \mathrm{s}$ was found to give the maximum pulse energy of the first amplified pulse and in this case the total energy of the output amplified seed burst was $100.1 \pm 0.2 \mathrm{~mJ}$. The output burst energy along with the digitized photodiode traces was used to calculate the pulse energies of the 5842 pulses shown in Fig. 5. These show a maximum pulse energy of $165.8 \pm 0.4 \mu \mathrm{J}$ with an exponential decay in pulse energy to a steady state. The mean pulse energy from pulse number 200 to 5000 is $17.0 \pm 0.6 \mu \mathrm{J}$ with a pulse to pulse variation of $0.5 \%$. The linear decrease in pulse energy at the end of the pulse train is indicative of the pump turn off and depletion of gain in the PCF. The high initial pulse energies and following exponential decay are shown more clearly in Fig. 6, which shows the first 36 pulses.

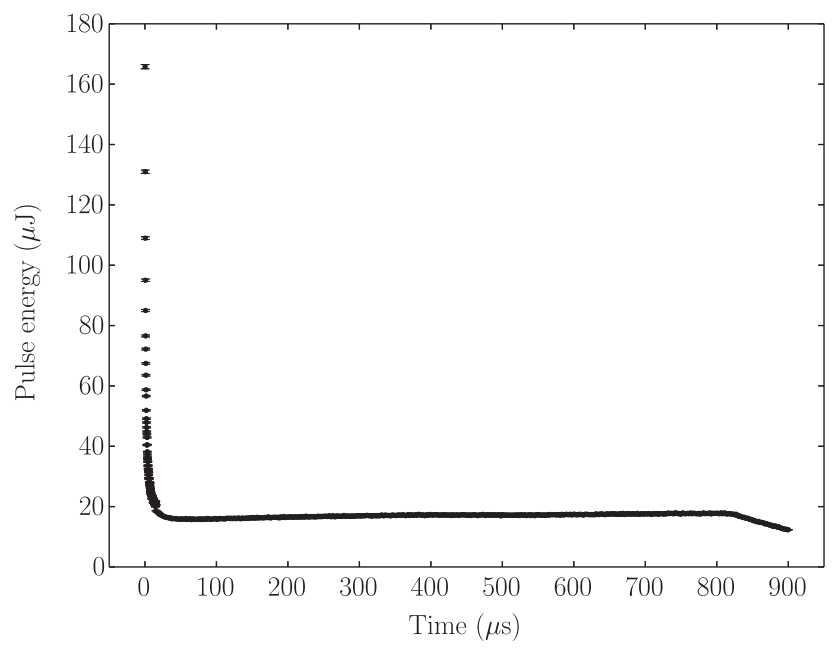

FIG. 5. Amplified pulse energies for a seed delay of $200 \mu \mathrm{s}$ relative to the pump.
To investigate the relationship between the delay of the seed burst with respect to the pump pulse and the energy of the first pulse in the amplified seed burst, the seed delay was varied from 0 to $200 \mu \mathrm{s}$ and the pulse energies of the full burst measured. The energy of the first pulse in the burst as a function of seed burst delay is shown in Fig. 7 .

With the simultaneous start of the seed and pump, the energy of the first pulse in the output burst was $0.71 \pm 0.01 \mu \mathrm{J}$, which is lower than the incident pulse energy from the seed of $0.95 \pm 0.01 \mu \mathrm{J}$. At a seed delay of $200 \mu \mathrm{s}$, the gain of the initial pulse was $27.2 \pm 0.1 \mathrm{~dB} \mathrm{~m}^{-1}$.

The long term stability of the laser system was observed by making energy measurements over several periods of up to 12 hours, over which a variation of $<1 \%$ was observed.

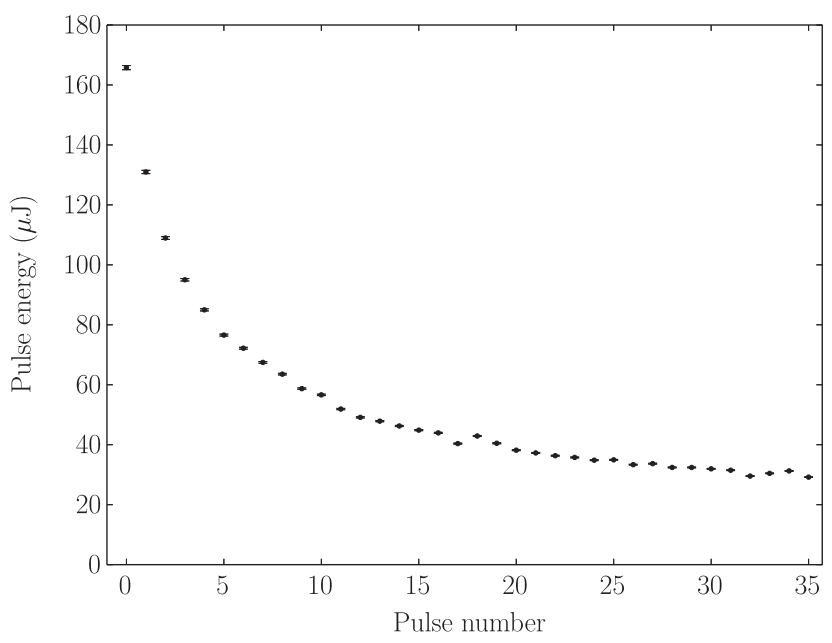

FIG. 6. Amplified pulse energies at the beginning of the seed burst shown in Fig. 5.

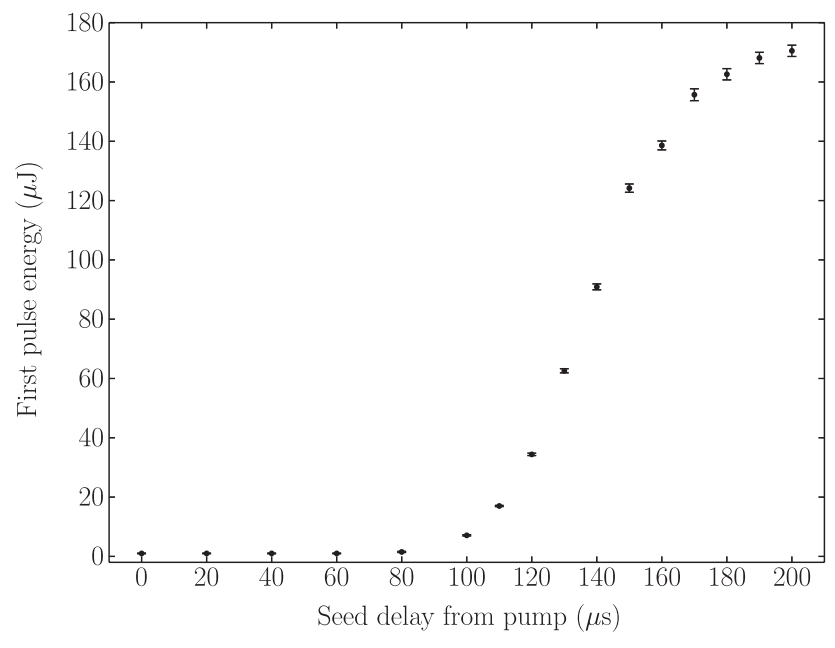

FIG. 7. Energy of the first pulse in the amplified train for various seed burst delays relative to the pump burst. 


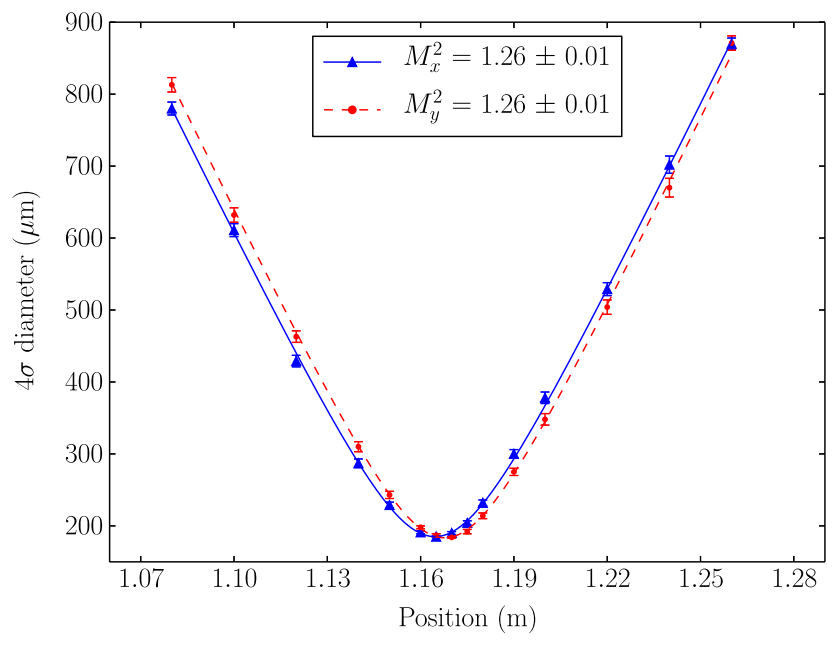

FIG. 8. $M^{2}$ measurement of amplified seed pulses with both the $x$ and $y$ fits shown.

\section{Spatial quality}

The spatial quality was measured by focusing the maximum amplified output beam from the PCF using an $f=1 \mathrm{~m}$ plano-convex lens. A laser beam profiler on a translation stage was used to measure the transverse profile at several points throughout the focus. The $4 \sigma$ diameter of the laser beam as it propagates can then be modeled by comparing it to a perfect Gaussian mode using the $M^{2}$ model [22]. The $M^{2}$ parameter of the laser beam then linearly scales the focused spot size for a given input beam size to a lens and its focal length, with a perfect Gaussian by definition having an $M^{2}$ of 1 . The $4 \sigma$ diameters and the $M^{2}$ model fits are shown in Fig. 8 with measured values of $M_{x}^{2}=M_{y}^{2}=1.26 \pm 0.01$. This excellent spatial quality is well suited for creating diffraction limited focused spot sizes.

\section{Pulse duration}

In a chirped pulse amplification laser system, the pulses are temporally stretched before amplification to avoid optical nonlinearities as well as damage to the amplifier and compressed back to their original duration afterwards. The stretcher introduces linear group delay dispersion (second order phase) to the pulse, stretching it in time, and the compressor is designed to compensate for this as well as any higher order phase terms accumulated by the pulse during amplification. However, dispersion caused by nonlinear effects such as self-phase modulation cannot necessarily be corrected and in this case the compressed pulse will have a different temporal profile and duration to the initial pulse. Although high peak powers can be achieved in optical fiber amplifiers, the presence of nonlinearities limits the compression and therefore the final peak power attainable. It is therefore important to measure the compressed pulse duration.

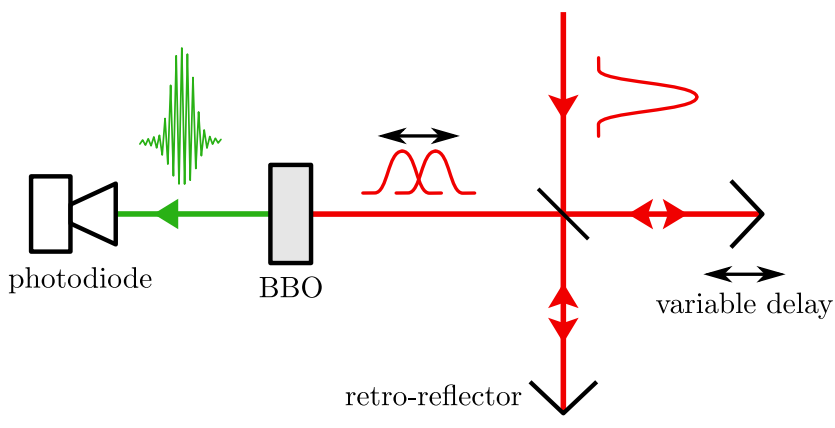

FIG. 9. Schematic of an autocorrelator, consisting of an interferometer with beam splitter, retroreflectors, a variable delay arm, BBO crystal and photodiode.

Ultrashort laser pulses well below 1 ns cannot be characterized by electronic devices alone as their response is too slow and therefore another technique must be used. Optical autocorrelation is the most common way of measuring these pulse durations [23] and therefore an autocorrelator based on a scanning Michelson interferometer was used for our pulse duration measurement. This consists of a 50:50 beam splitter to split the incoming pulse, two retroreflectors to recombine the split pulses at the beam splitter and a beta-barium borate $\left(\beta-\mathrm{BaB}_{2} \mathrm{O}_{4}\right.$, $\mathrm{BBO})$ frequency doubling crystal to analyze the interference between the two pulses as shown in Fig. 9. One arm of the interferometer is mounted on a translation stage and as it is scanned the delay between the two pulses is varied. The resultant frequency doubled output as detected by a photodiode shows the interference fringes, the envelope of which is the convolution of the pulse with itself. The standard technique involves scanning one arm of the interferometer and continuously recording data. In our case however, the low duty cycle of the bursts and the significant time taken to digitize the waveforms makes this implementation unsuitable. Instead, waveforms were recorded at a series of static positions and the setup was mechanically agitated during the data recording, allowing the envelope to be observed at that position. A large number of waveforms were recorded at each position to ensure the full extent of the envelope was captured. To deconvolve the autocorrelation an assumption about the temporal profile of the pulse is required. We assume a Gaussian temporal profile with a deconvolution factor of $\sqrt{2}$.

For verification, we compared pulse duration measurements made with both the method described above and also the conventional scanning autocorrelation technique. To perform this comparison, the EOM was turned off so that the laser output consisted of a series of continuous pulses rather than operating in a burst mode. This enabled us to take data using both methods that could be directly compared. The conventional scanning autocorrelation is shown in Fig. 10. The upper envelopes of this autocorrelation and that of the autocorrelation performed in burst 


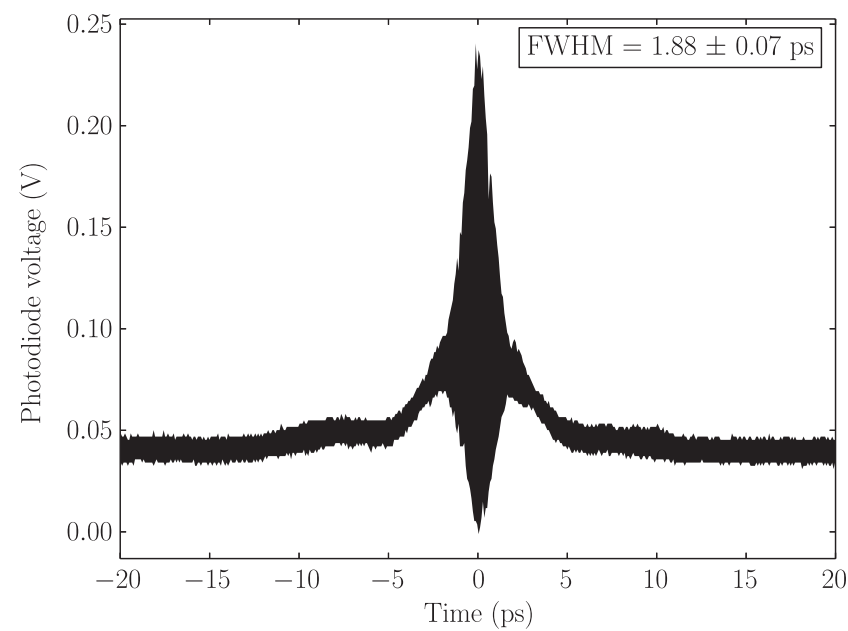

FIG. 10. Autocorrelation of the seed laser compressed without transmission through the final PCF amplification stage.

mode are shown together in Fig. 11 and show excellent agreement between both methods with a deconvolved full width at half maximum (FWHM) of $1.88 \pm 0.07 \mathrm{ps}$ in both cases. This is also indicative of the achievable pulse durations possible from this seed laser used with the PCF.

Having measured the compressed output of the commercial system before the PCF, we then measured the effect of transmission through the PCF both with and without amplification. The autocorrelation of the unamplified compressed output from the PCF is shown in Fig. 12, which shows a slightly wider peak with a deconvolved FWHM of $3.85 \pm 0.13 \mathrm{ps}$ as well as slight wings. The transmission through the PCF introduces additional dispersion to the pulse that could not be fully compensated for by the compressor of the commercial laser used in this research,

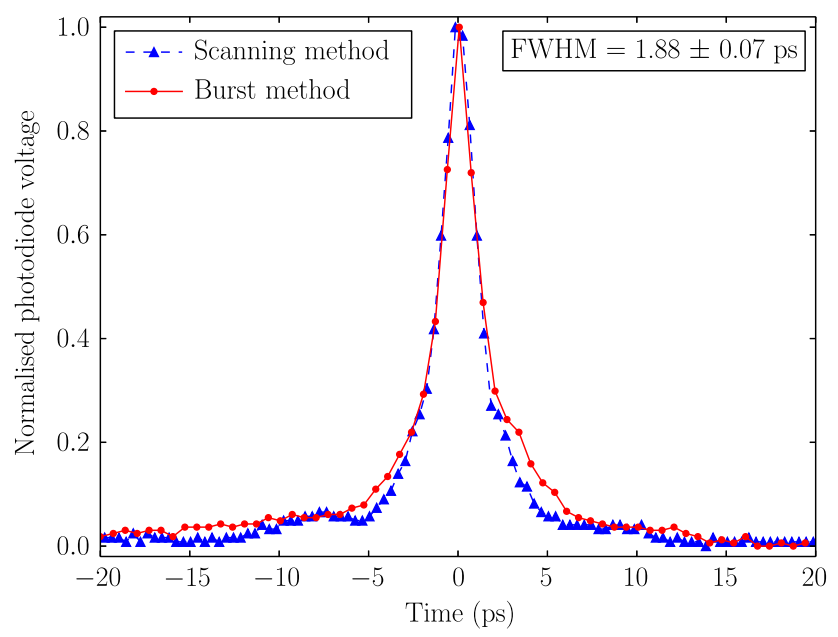

FIG. 11. Envelope of autocorrelations of the seed laser made using both the conventional scanning method and the burst operation method showing good agreement with identical FWHM.

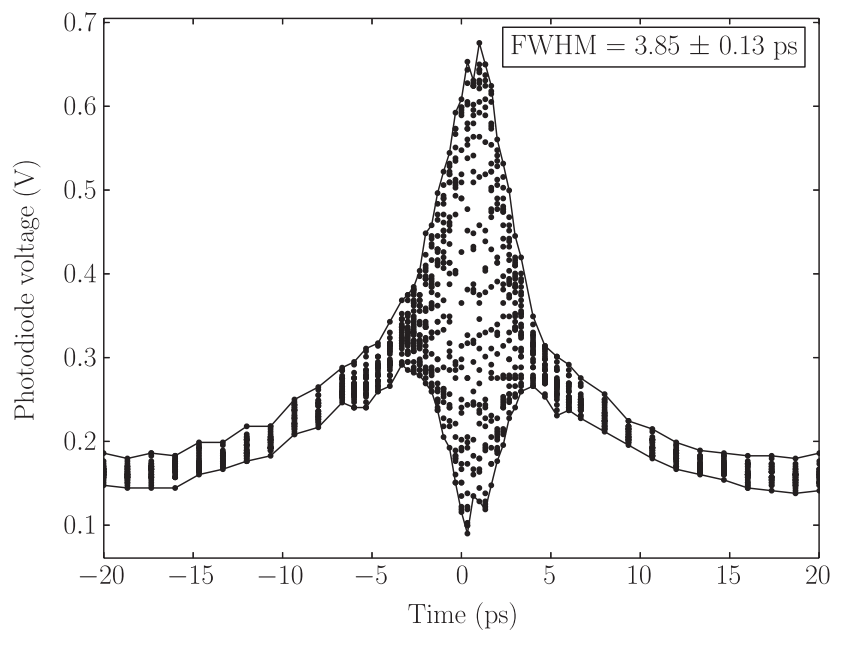

FIG. 12. Autocorrelation of the unamplified compressed seed pulses transmitted through the PCF.

which has a fixed layout and a transmission efficiency of $75 \%$.

Figure 13 shows the autocorrelation of the first pulse in the amplified burst with the highest energy and therefore the most likely to have generated optical nonlinearities. In this case, only a small fraction of the amplified output from the PCF was compressed to avoid damage to the commercial compressor. The autocorrelation shows a more complicated structure than the previous autocorrelations with a narrow peak with a FWHM of $1.92 \pm 0.12$ ps as well as double wings on either side. Again, the fixed compressor arrangement prevents the possible compensation of additional dispersion introduced by the amplification. The wings in the autocorrelation indicate a temporal structure beyond a simple Gaussian such as a prepulse or postpulse [24]. The central peak, as defined by the minima on either

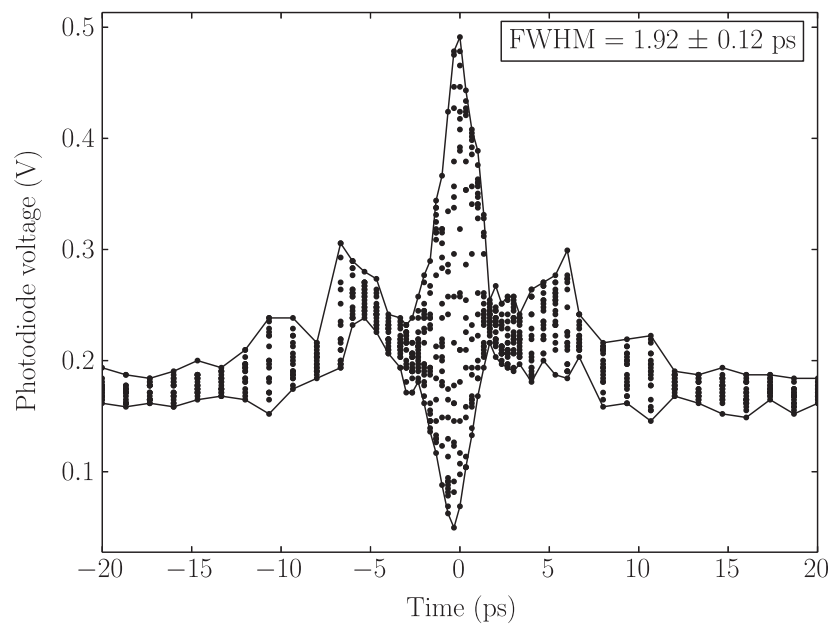

FIG. 13. Autocorrelation of the first pulse in the amplified burst with an energy of $165.8 \pm 0.4 \mu \mathrm{J}$ with the envelope shown. 


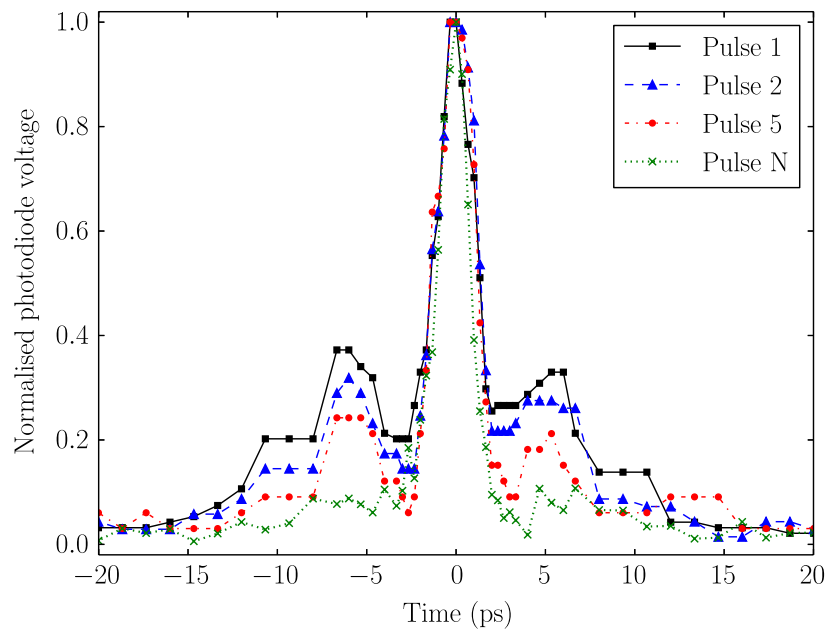

FIG. 14. Autocorrelation envelopes of pulses 1,2, 5 and the $n$th pulse, which is a pulse much later in the burst that is in the steady state. Each is normalized showing a clear reduction in the wings as the pulse energy decreases.

side, accounts for $40 \pm 1 \%$ of the total area of the autocorrelation and therefore that percentage of the total pulse energy is contained within the central peak. With the initial pulse energy of $165.8 \pm 0.4 \mu \mathrm{J}$, and the compressor transmission of $75 \%$, the peak power is correspondingly $25.8 \pm 1.7 \mathrm{MW}$. The normalized upper envelopes of the autocorrelations of this and subsequent pulses are shown in Fig. 14.

It can be seen that for pulses further into the train, the wings are smaller in the autocorrelations and a higher percentage of the pulse energy is contained within the central peak. As the pulses are also decreasing in energy, this would indicate an intensity dependence consistent with the presence of optical nonlinearities. The peak powers calculated from the percentage of energy in the central peak and the pulse durations are shown in Table II. These peak powers would be significantly enhanced by the use of a compressor properly designed to compensate for the phase accumulated in the PCF amplifier.

\section{Polarization}

As discussed in Sec. I, the infrared laser source must be frequency doubled to the visible part of the spectrum to achieve a smaller focused spot size. Frequency conversion requires that the input source be highly linearly polarized. Optical fibers do not preserve the polarization of an input source due to inhomogeneities and stresses on the fiber caused by bending and torsion along its length. However, unlike most optical fibers the rod-type PCF is straight and therefore these effects were expected to be minimal.

The degree of linear polarization (DOLP) was measured by recording the transmitted power through a polarizing beam splitter as a function of angle and the maximum, $P_{\max }$ and minimum, $P_{\min }$ transmitted powers are used to determine the DOLP using Eq. (2):

$$
\operatorname{DOLP}(\%)=100 \times\left(\frac{P_{\max }-P_{\min }}{P_{\max }+P_{\min }}\right) .
$$

The DOLP of the input seed was measured to be $96.4 \% \pm 0.4 \%$ and the DOLP of the amplified output was $95.3 \% \pm 0.3 \%$. This shows that the output is linearly polarized and that amplification in the PCF did not affect the polarization despite the lack of specific polarization maintaining structure.

\section{Pointing stability}

The laser system for a laserwire cannot be placed next to the accelerator beam line directly due to the damaging high radiation environment. Furthermore, this placement would preclude access to the laser system for maintenance. Therefore, the laser system must be located in a separate area from the particle accelerator and the laser beam transported to the laserwire stations by either a system of mirrors (free-space transport) or in fiber. With the required peak powers, it is not currently possible to transport laser pulses in fiber without incurring optical nonlinearities that would distort the temporal and spectral properties of the laser pulse.

TABLE II. Summary of the measured properties and calculated peak powers of the pulses using the compressor transmission efficiency of $75 \%$. The $n$th pulse is representative of the mean of the steady-state pulses. The number of interacting photons $\left(N_{\gamma \text { interacting }}\right)$ was calculated by calculating the proportion of the total pulse energy within the full width at half maximum (FWHM) of the central peak. This was scaled assuming 75\% compressor transmission and $60 \%$ frequency doubling efficiency. The number of Comptonscattered photons is calculated from this assuming ILC electron beam parameters detailed in Sec. IV for two possible laserwire collision angles of $\pi / 2$ and $\pi / 6$.

\begin{tabular}{lccccccc}
\hline \hline Pulse number & Energy $\mu \mathrm{J}$ & FWHM ps & Central peak $\%$ & Peak power MW & $N_{\gamma \text { interacting }}$ & $N_{C}(\theta=\pi / 2)$ & $N_{C}(\theta=\pi / 6)$ \\
\hline 1 & $165.8 \pm 0.4$ & $1.92 \pm 0.12$ & 40 & $25.8 \pm 1.7$ & $7.8 \times 10^{13}$ & 8496 & 22495 \\
2 & $131.0 \pm 0.4$ & $1.89 \pm 0.12$ & 40 & $20.8 \pm 1.4$ & $6.1 \times 10^{13}$ & 6755 \\
3 & $109.0 \pm 0.4$ & $1.82 \pm 0.11$ & 41 & $18.5 \pm 1.2$ & $5.2 \times 10^{13}$ & 5845 & 17884 \\
4 & $95.1 \pm 0.3$ & $1.72 \pm 0.11$ & 45 & $18.6 \pm 1.3$ & $5.0 \times 10^{13}$ & 5712 & 15472 \\
5 & $84.9 \pm 0.3$ & $1.75 \pm 0.11$ & 54 & $19.9 \pm 1.4$ & $5.4 \times 10^{13}$ & 6082 & 16118 \\
$n$th & $17.0 \pm 0.1$ & $1.73 \pm 0.11$ & 100 & $7.4 \pm 0.5$ & $2.0 \times 10^{13}$ & 2265 & 5994 \\
\hline \hline
\end{tabular}


Under free-space propagation, the pointing stability of the laser must be considered as the angular jitter of the laser beam produces spatial jitter of the laser profile at the laserwire lens. As many laser pulses are required to make a laserwire scan, the corresponding spatial jitter of the focused laser beam is therefore convolved with the transverse size of the electron beam and increases the measured transverse size.

The pointing stability of the laser system was measured by measuring the laser profile centroid of the collimated output laser beam using a high resolution CCD beam profiler at a distance of $3.518 \mathrm{~m}$, where the laser beam size $(4 \sigma)$ was measured to be $8.157 \pm 0.022 \mathrm{~mm}$. The standard deviation of the centroid was measured over a $20 \mathrm{~min}$ period to be $3.820 \mu \mathrm{m}$, which corresponds to an angular jitter of $1.09 \pm 0.03 \mu \mathrm{rad}$. For a laserwire system at a distance of $20 \mathrm{~m} \mathrm{[25]} \mathrm{producing} \mathrm{a} 1 \mu \mathrm{m}$ focused spot size using this laser beam, the spatial jitter at the focus would be $<10 \mathrm{~nm}$. When convolved with the laserwire scan this contribution would be negligible. At a greater distance, the contribution may be non-negligible, but measurable and can be subtracted in analysis.

\section{APPLICATION AS A LASERWIRE}

In order to assess the suitability of the developed laser system for a laserwire, the yield of Compton-scattered photons from a laser pulse with an electron bunch must be considered along with the detector efficiency and required precision. Reviews of luminosity calculations and Compton-scattering cross sections are given in $[4,26]$ and a brief summary is given here.

The number of Compton-scattered photons is given by the product of the luminosity $L(\theta)$ and the Compton cross section $\sigma_{C}(\omega)$, which are dependent on the collision angle $[27,28]$. The Compton cross section is given by the product of the Thomson cross section $\left(\sigma_{T}=0.665 \times 10^{-28} \mathrm{~m}^{2}\right)$ with $f(\omega)$, which is given by

$$
\begin{aligned}
f(\omega)= & \frac{3}{4}\left\{\frac{1+\omega}{\omega^{3}}\left[\frac{2 \omega(1+\omega)}{1+2 \omega}-\ln (1+2 \omega)\right]\right. \\
& \left.+\frac{\ln (1+2 \omega)}{2 \omega}-\frac{1+3 \omega}{(1+2 \omega)^{2}}\right\},
\end{aligned}
$$

where $\omega$ is the normalized energy of the laser photons in the electron rest frame, is given by

$$
\omega=\frac{h E_{b}}{\lambda c^{3} m_{e}^{2}}(1+\cos \theta) .
$$

Here, $\lambda$ is the laser wavelength and $E_{b}$ the electron beam energy ( $h$ is the Planck constant, $c$ the speed of light and $m_{e}$ the electron mass). The luminosity $L(\theta)$ can be decomposed into the product of the luminosity for a head-on collision $L(0)$ and a reduction factor $R(\theta)$,

$$
\begin{gathered}
L(0)=\frac{n_{\gamma} n_{e}}{2 \pi \sqrt{\sigma_{\gamma x}^{2}+\sigma_{e x}^{2}} \sqrt{\sigma_{\gamma y}^{2}+\sigma_{e y}^{2}}}, \\
R(\theta)=\frac{1}{\sqrt{1+\left(\frac{\sigma_{\gamma z}^{2}+\sigma_{e z}^{2}}{\sigma_{\gamma x}^{2}+\sigma_{e x}^{2}}\right) \tan ^{2}(\theta / 2)}},
\end{gathered}
$$

where $n_{\gamma}$ and $n_{e}$ are the number of photons in the laser pulse and electrons in a bunch respectively. The laser pulse and electron bunch are assumed to have Gaussian distributions with the corresponding sigma parameters in three orthogonal dimensions; $\sigma_{\gamma x}, \sigma_{\gamma y}, \sigma_{\gamma z}$ and $\sigma_{e x}, \sigma_{e y}, \sigma_{e z}$ respectively. For simplicity, the laser divergence is neglected, i.e., it is assumed that the Rayleigh range of the laser is large compared to $\sigma_{e x}$.

Taking the ILC as an example, representative parameters of the electron beam in the BDS part of the accelerator where a laserwire is likely to be used are $E_{b}=250 \mathrm{GeV}$, $n_{e}=2 \times 10^{10}, \quad \sigma_{e x}=10 \mu \mathrm{m}, \quad \sigma_{e y}=1 \mu \mathrm{m}$ and $\sigma_{e z}=$ $300 \mu \mathrm{m}$ [5]. Assuming 75\% transmission through a laser compressor, $60 \%$ frequency-doubling conversion efficiency $(\lambda=518 \mathrm{~nm})$, the measured pulse energies from the laser system described are used to estimate the number of Compton-scattered photons for collision angles of both $\pi / 2$ and $\pi / 6$ as summarized in Table II. Only the pulse energy within the central peak of the laser pulse is considered.

The collision angle of $\pi / 6$ in the horizontal plane increases the luminosity but effectively reduces the distance over which the laser beam diverges across the electron beam. With the electron beam sizes mentioned, the reduced Rayleigh range is still over double the width of the electron beam. Even when present, such effects do not preclude the use of a laserwire as has been recently shown [25].

Assuming a detector efficiency of 5\% [5], the number of detected Compton-scattered photons from the highest energy pulse with an angle of $\pi / 2$ would be 425 , which is enough for a good electron beam emittance measurement [5]. With an angle of $\pi / 6$, this is increased to 1125 detected photons, which would significantly improve the detector statistical accuracy. Using the steady state pulse energy at this angle, 300 photons would be detected, which is still adequate for a laserwire at the ILC.

\section{CONCLUSIONS AND OUTLOOK}

A fiber laser system suitable for an ILC laserwire has been demonstrated. Using a novel burst mode amplification regime, pulses an order of magnitude greater in energy than achievable in the steady state have been shown with a maximum pulse energy of $165.8 \pm 0.4 \mu \mathrm{J}$. This method was shown to be controllable by varying the relative timing of the pump and the seed bursts allowing a range of initial pulse energies. The methods developed to characterize the $6.49 \mathrm{MHz}$ pulses were detailed, which allowed 
measurement of the pulse energy and autocorrelation on a pulse by pulse basis. The spatial quality of the amplified laser output was measured and shown to be excellent, which would allow small focused spot sizes to be achieved.

The demonstrated high peak powers could be further improved with a specifically designed compressor that properly compensates for the additional group delay dispersion introduced in the PCF to produce shorter duration pulses and consequently higher peak powers. In this case, the useful pulse energy within the pulse duration would be significantly increased from $40 \%$ demonstrated in the highest energy pulse. Furthermore, stretching the oscillator pulses to approximately $1 \mathrm{~ns}$ is possible and would significantly reduce the nonlinearities accumulated. Research on better compression and more efficient gain extraction in the PCF is under way.

Regarding the use of the laser for a laserwire diagnostic, the system described would currently be suitable for use with the ILC and if required, averaging of multiple electron bunches would decrease the uncertainty of the measurement. The demonstrated laser pulse train varies significantly in pulse energy with an initial pulse approximately an order of magnitude greater than the steady state. Normalization could be used to account for this variation as the pattern was found to be very stable. Whilst the steady state pulse energies are sufficient, it may be highly desirable to have a different operational mode for a laserwire where a small number of high energy pulses can be generated allowing alignment or calibration.

The background conditions for such an accelerator have yet to be accurately simulated and this is an active area of research. With this information, the required laser pulse energy could be more precisely defined. Simulation of the laserwire signal in combination with the accelerator backgrounds is also highly relevant as although laserwire diagnostics have been demonstrated at test facilities, the high energy case of the ILC will provide multi-GeV Compton-scattered photons that may be more distinguishable from background sources than those at the lower energy test facilities. In addition, improvement of the detector efficiency would have a significant impact on the required laser peak power.

This system demonstrates the peak powers required for a laserwire diagnostic as well as excellent spatial quality, which in addition to the high electrical efficiency of fiber lasers demonstrates their suitability for accelerator applications.

\section{ACKNOWLEDGMENTS}

The research leading to these results has received funding from the Science and Technology Facilities Council via the John Adams Institute, University of Oxford and CERN.
[1] C. Adolphsen, M. Barone, B. Barish, K. Buesser, P. Burrows et al., arXiv:1306.6328.

[2] M. Aicheler, P. Burrows, M. Draper, T. Garvey, P. Lebrun, K. Peach, and N. Phinney, "A Multi-TeV Linear Collider Based on CLIC Technology: CLIC Conceptual Design Report," 2012 (unpublished).

[3] D. Brandt, "CERN Accelerator School: Course on Beam Diagnostics," 2009 (unpublished).

[4] P. Tenenbaum and T. Shintake, Annu. Rev. Nucl. Part. Sci. 49, 125 (1999).

[5] I. Agapov, G. Blair, and M. Woodley, Phys. Rev. ST Accel. Beams 10, 112801 (2007).

[6] R. Alley, D. Arnett, E. Bong, W. Colocho, J. Frisch, S. Horton-Smith, W. Inman, K. Jobe, T. Kotseroglou, D. McCormick, J. Nelson, M. Scheeff, S. Wagner, and M. Ross, Nucl. Instrum. Methods Phys. Res., Sect. A 379, 363 (1996).

[7] M. Ross, in Proceedings of the 2003 Particle Accelerator Conference, Portland, OR (IEEE, New York, 2003), Vol. 1, pp. 503-507.

[8] S. Boogert, G. Blair, G. Boorman, A. Bosco, L. Deacon, P. Karataev, A. Aryshev, M. Fukuda, N. Terunuma, J. Urakawa, L. Corner, N. Delerue, B. Foster, D. Howell, M. Newman, R. Senanayake, R. Walczak, and F. Ganaway, Phys. Rev. ST Accel. Beams 13, 122801 (2010).

[9] T. Aumeyr, G. Blair, S. Boogert, G. Boorman, A. Bosco, K. Balewski, E. Elsen, V. Gharibyan, G. Kube, S. Schreiber, and K. Wittenburg, in Proceedings of the International Particle Accelerator Conference, Kyoto, Japan (ICR, Kyoto, 2010), MOPE069.

[10] H. Sakai, Y. Honda, N. Sasao, S. Araki, Y. Higashi, T. Okugi, T. Taniguchi, J. Urakawa, and M. Takano, Phys. Rev. ST Accel. Beams 4, 022801 (2001).

[11] L. Nevay, G. Blair, S. Boogert, P. Karataev, K. Kruchinin, L. Corner, and R. Walczak, in Proceedings of the International Particle Accelerator Conference, Shanghai, China (JaCoW, Shanghai, China, 2013), MOPWA053.

[12] J. Rothhardt, T. Eidam, S. Hädrich, F. Jansen, F. Stutzki, T. Gottschall, T. V. Andersen, J. Limpert, and A. Tünnermann, Opt. Lett. 36, 316 (2011).

[13] D. J. Richardson, J. Nilsson, and W. A. Clarkson, J. Opt. Soc. Am. B 27, B63 (2010).

[14] J. Limpert, R. Fabian, D. N. Schimpf, E. Seise, T. Eidam, H. Steffen, and J. Rothhardt, IEEE J. Quantum Electron. 15, 159 (2009).

[15] J. Limpert, N. Deguil-Robin, I. Manek-Hönninger, F. Salin, F. Röser, A. Liem, T. Schreiber, S. Nolte, H. Zellmer, A. Tünnermann, J. Broeng, A. Petersson, and C. Jakobsen, Opt. Express 13, 1055 (2005).

[16] C. D. Brooks and F. Di Teodoro, Appl. Phys. Lett. 89, 111119 (2006).

[17] F. Röser, T. Eidam, J. Rothhardt, O. Schmidt, D. N. Schimpf, J. Limpert, and A. Tünnermann, Opt. Lett. 32, 3495 (2007).

[18] D. Xue, Q. Lou, and J. Zhou, Opt. Laser Technol. 39, 871 (2007).

[19] J. W. Dawson, M. J. Messerly, R. J. Beach, M. Y. Shverdin, E. A. Stappaerts, A. K. Sridharan, P. H. Pax, J. E. Heebner, C. W. Siders, and C. Barty, Opt. Express 16, 13240 (2008). 
[20] J. Limpert, N. Deguil-Robin, S. Petit, I. Manek-Hönninger, F. Salin, P. Rigail, C. Hönninger, and E. Mottay, Appl. Phys. B 81, 19 (2005).

[21] S. Breitkopf, A. Klenke, T. Gottschall, H. J. Otto, C. Jauregui, J. Limpert, and A. Tünnermann, Opt. Lett. 37, 5169 (2012).

[22] T. Johnston, Appl. Opt. 37, 4840 (1998).

[23] A. Monmayrant, S. Weber, and B. Chatel, J. Phys. B 43, 103001 (2010).
[24] R. Trebino, C. C. Hayden, A. M. Johnson, W. M. Simpson, and A. M. Levine, Opt. Lett. 15, 1079 (1990).

[25] L. Nevay, S. Boogert et al., Phys. Rev. ST Accel. Beams 10, 072802 (2014).

[26] T. Suzuki, KEK Technical Report No. KEK-76-3, 1976.

[27] O. Klein and Y. Nishina, Z. Phys. 52, 853 (1929).

[28] Y. Miyahara, Nucl. Instrum. Methods Phys. Res., Sect. A 588, 323 (2008). 